
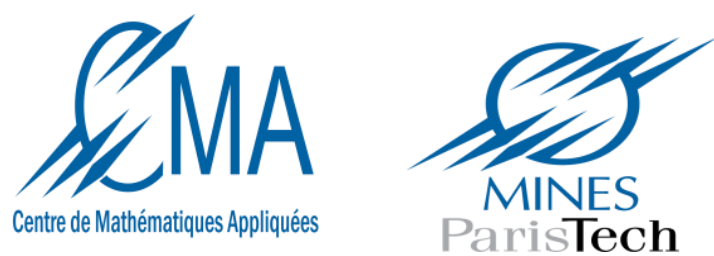

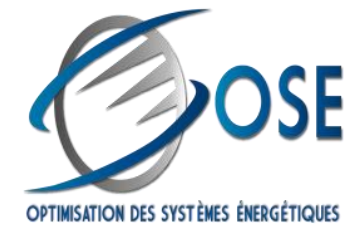

PSL $\star$

RESEARCH UNIVERSITY PARIS

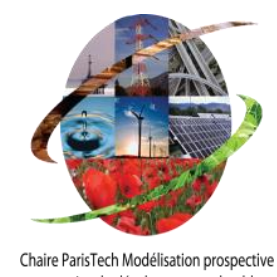

\title{
A stochastic optimization model for frequency control and energy
} management in a microgrid

Dhekra Bousnina (CMA, Mines ParisTech, France)

Welington de Oliveira (CMA, Mines ParisTech, France)

Peter Pflaum (Schneider-Electric, Analytics Applications and Programs, France) 


\section{Agenda}

1 Context of the work

2 Problem formulation

3 Out-of-sample simulations

$4 \quad$ Closed-loop simulations

5 Conclusions \& future work

Page 2 
The microgrid system description:

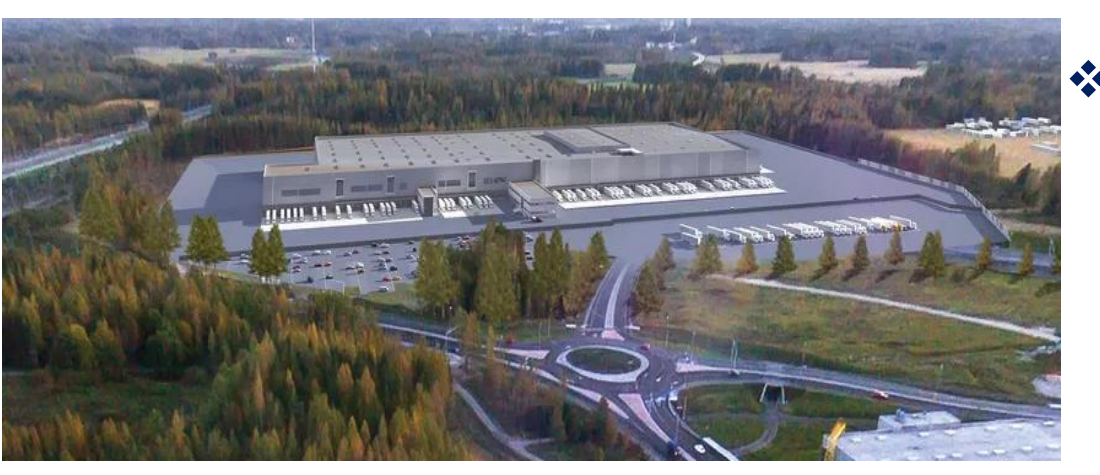

A thermal-electrical microgrid in Finland
- Participating to the Frequency Containment Reserve market
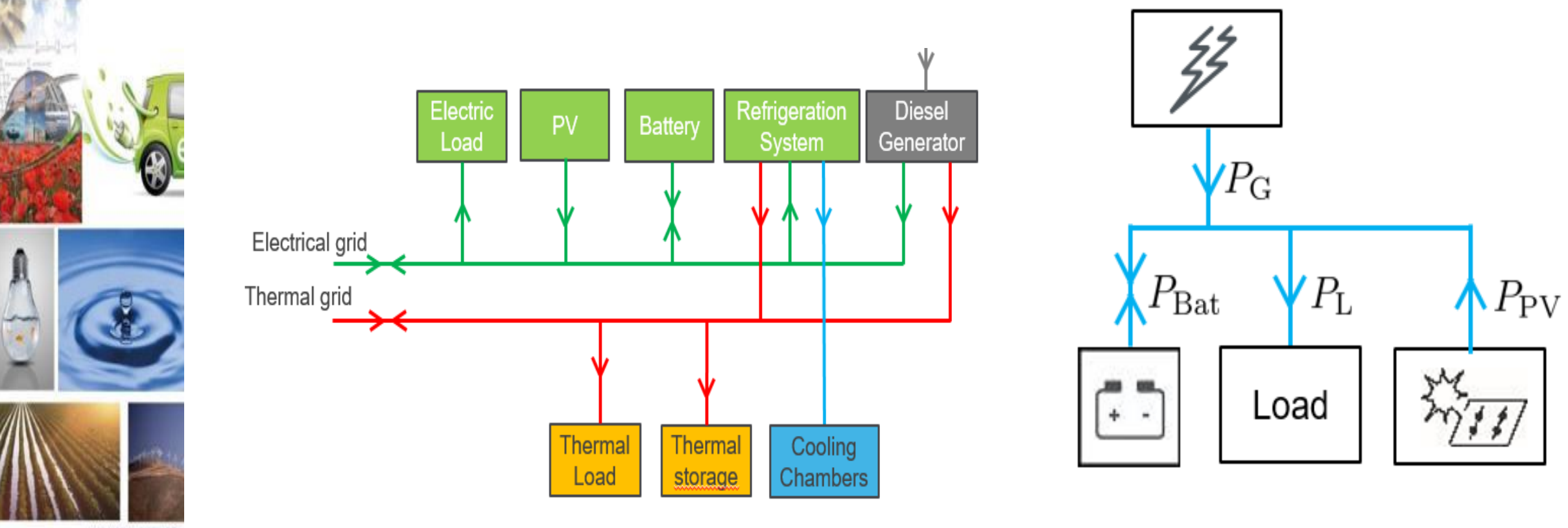

Page 3 Mive pyHtf 


\section{Frequency Containement Reserve: requirements}

* When $\mathbf{P}_{\text {produced }} \neq \mathbf{P}_{\text {consumed }}$ : Frequency of the grid deviated from its nominal value $(\mathbf{5 0} \mathbf{~ H z})$

* Need for frequency regulation to compensate deviations

* Any producer/consumer of a sufficient size (> 100kW) can participate in the FCR market

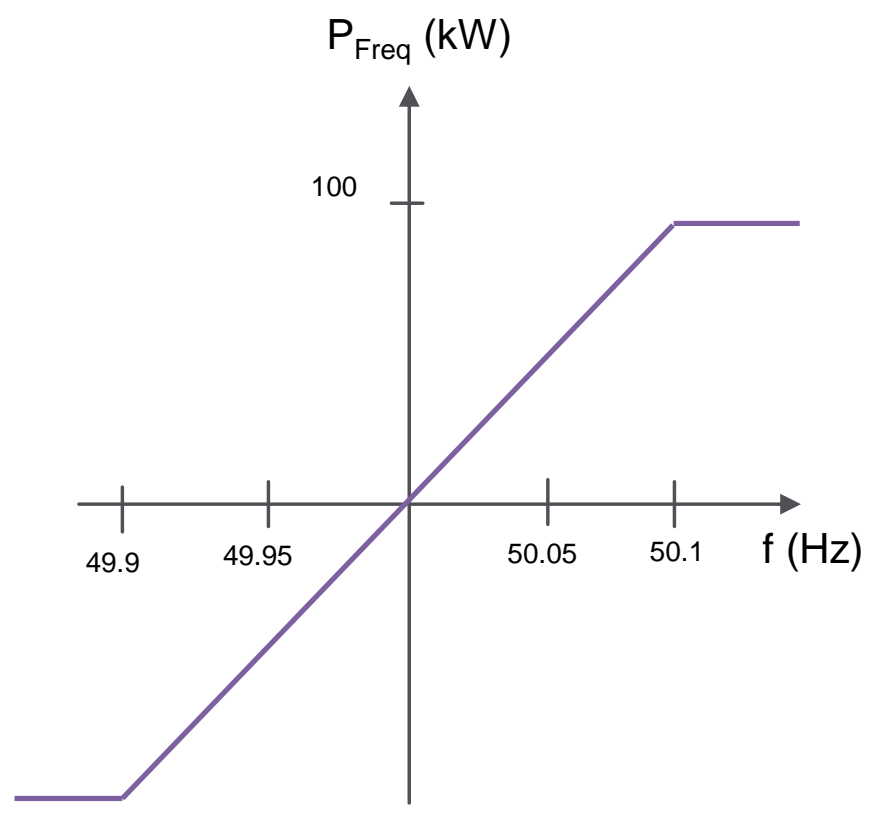




\section{Frequency Containement Reserve: problems}

-What is the optimal dayahead frequency reserve profile to be submitted?

Solve the planning optimization problem

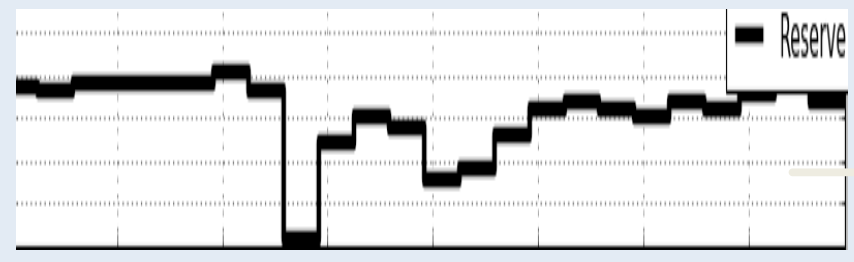

Day J-1
What is the the optimal control strategy for the battery to minimize energy costs?

Solve the control optimization problem

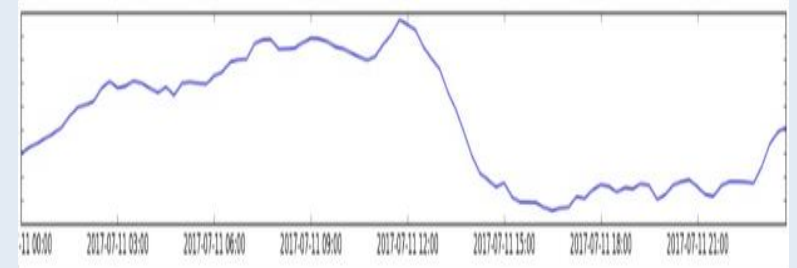

Day J 


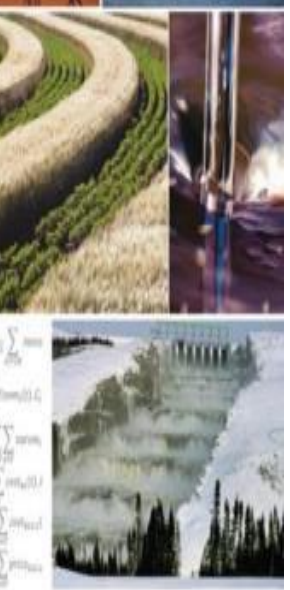

Example of historical frequency deviation realization
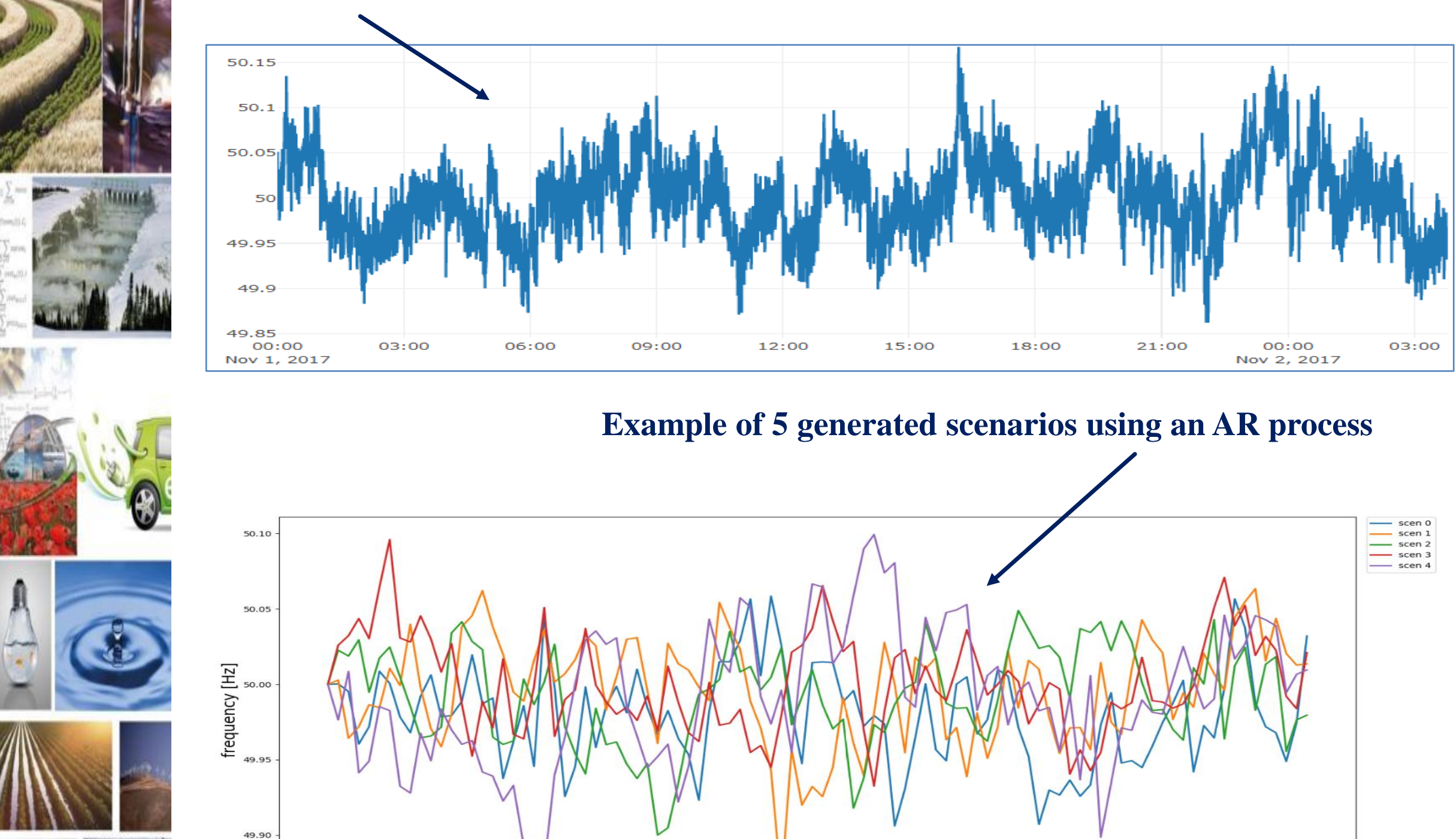

Page 6

Example of 5 generated scenarios using an AR process

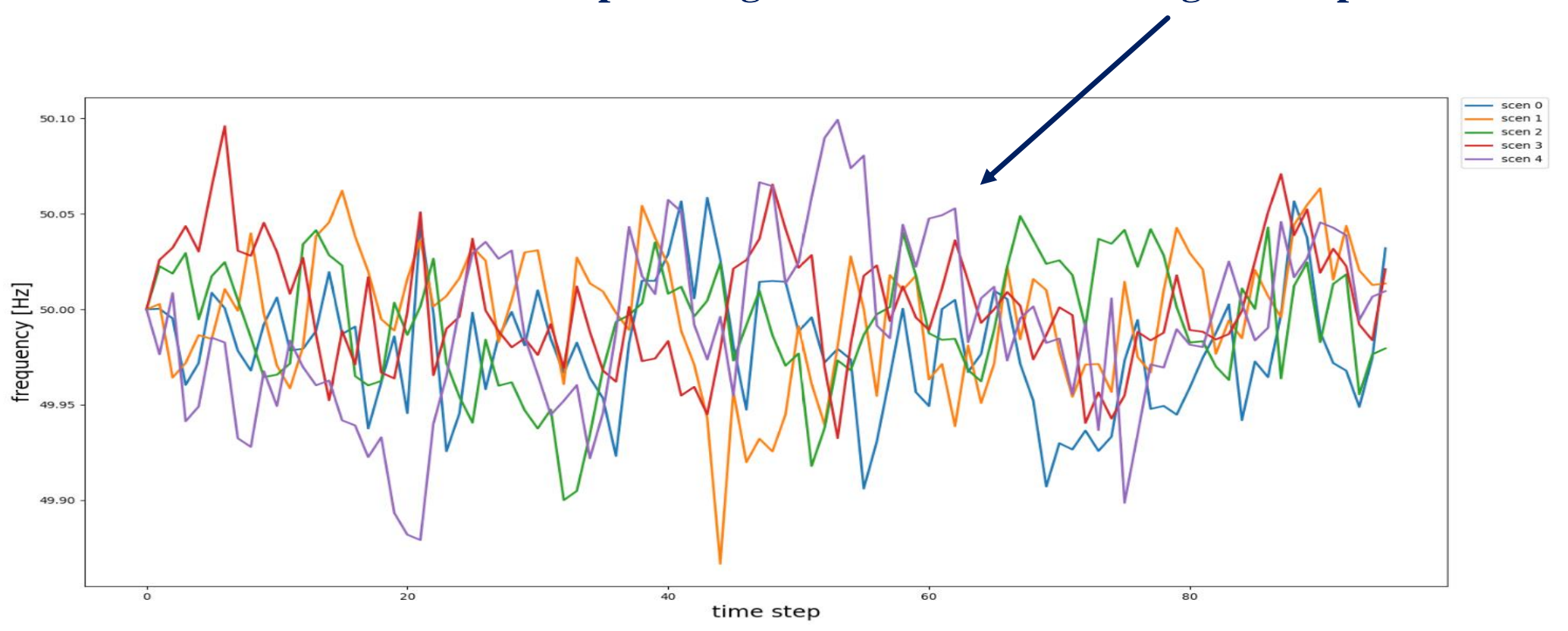




\section{Agenda}

1 Context of the work

\section{Problem formulation}

3 Out-of-sample simulations

$4 \quad$ Closed-loop simulations

5 Conclusions \& future work 


\section{Planning problem: deterministic}

Revenue from supplied Energy TOU costs FCR

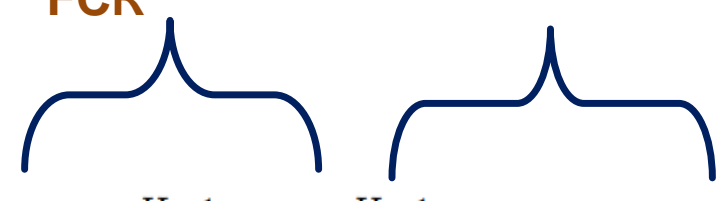

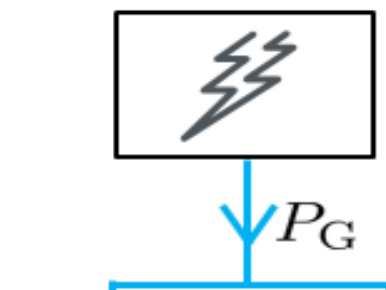

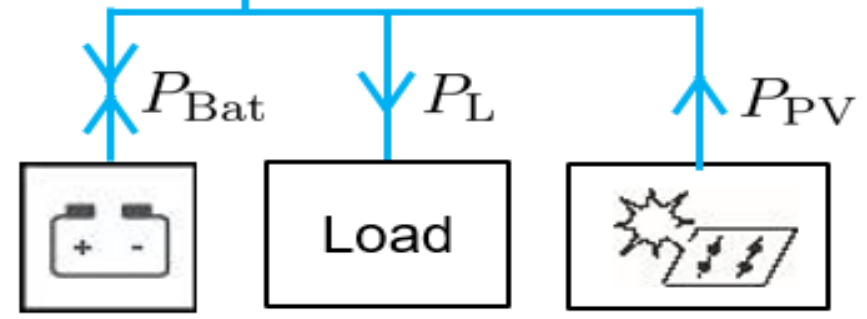

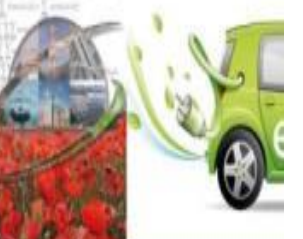

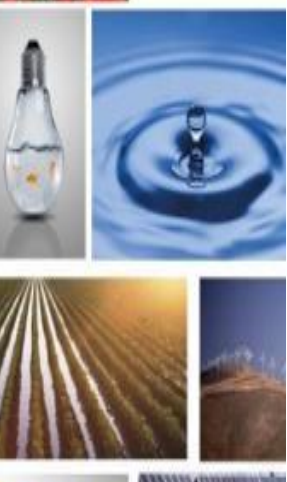$$
\min -C_{\mathrm{R}} \sum_{t=0}^{H-1} R_{t}+\sum_{t=0}^{H-1} C_{\mathrm{TOU}, t} P_{\mathrm{G}, t}
$$$$
\text { s.t. } \quad P_{\mathrm{G}, t}=P_{\mathrm{L}, t}+P_{\mathrm{B}, t}+P_{\mathrm{pv}, t} \quad \forall t
$$$$
S o C_{t+1}=S o C_{t}+P_{\mathrm{B}, t} . \Delta t . \eta
$$$$
P_{\text {min }}+R_{t} \leq P_{\mathrm{B}, t} \leq P_{\max }-R_{t}
$$$$
\forall t
$$$$
S o C_{\text {min }}+0.5 R_{t} \leq S o C_{t} \leq S o C_{\text {max }}-0.5 R_{t} \quad \forall t
$$

Balance equation

$R_{t} \geq 0 \quad \forall t$.
Battery State of Charge

Power and energy reserve for frequency regulation 


\section{Planning problem: stochastic}

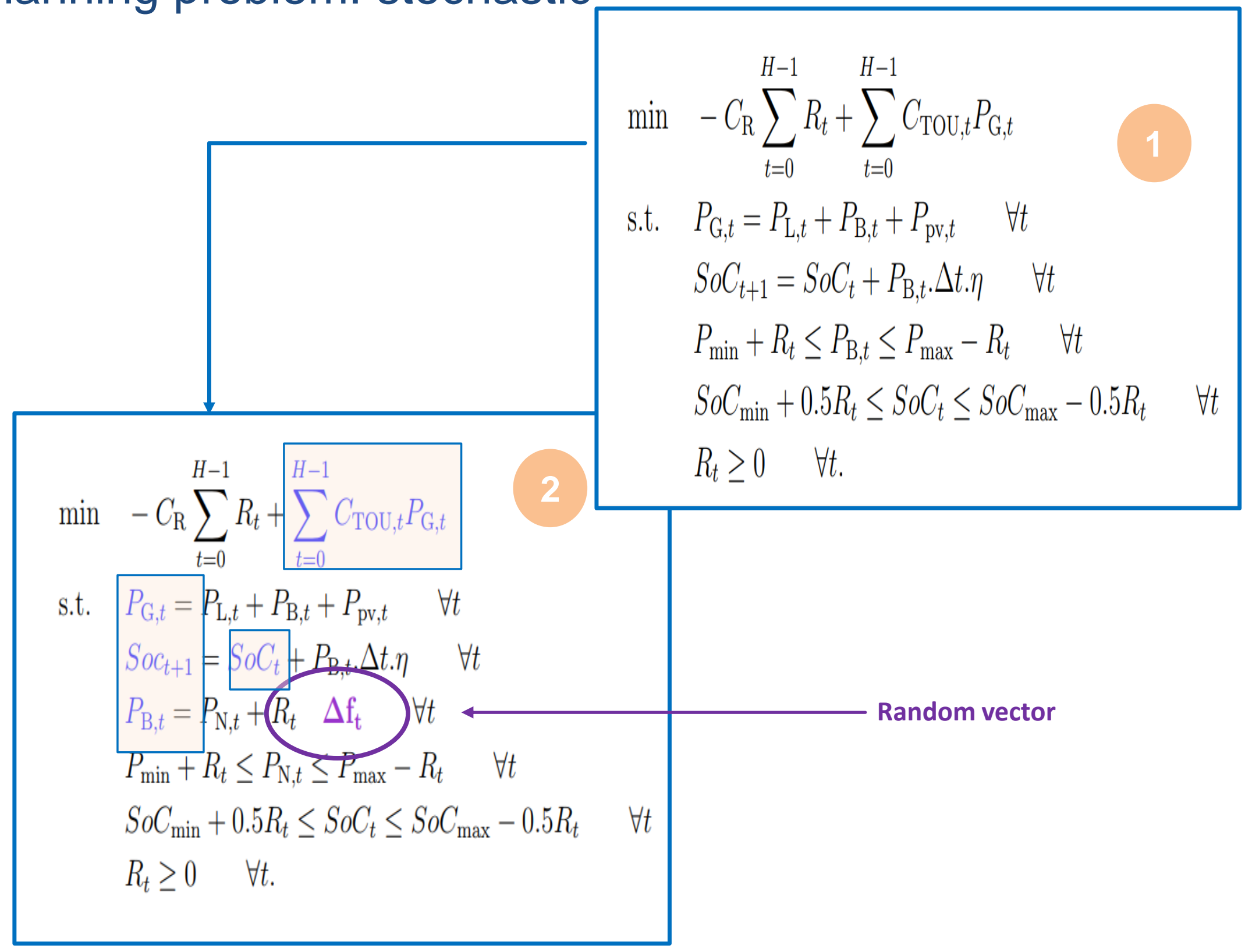




\section{Two-stage stochastic formulation:}

Two-stage stochastic formulation:

$\min -C_{\mathrm{R}} \sum_{t=0}^{H-1} R_{t}+\mathbb{E}\left[\sum_{\mathrm{t}=0}^{\mathrm{H}-1} \mathrm{C}_{\mathrm{TOU}, t} \mathrm{P}_{\mathrm{G}, \mathrm{t}}\right]$

s.t. $\quad P_{\mathrm{G}, t}=P_{\mathrm{L}, t}+P_{\mathrm{B}, t}+P_{\mathrm{pv}, t} \quad \forall t$ $S o c_{t+1}=S o C_{t}+P_{\mathrm{B}, t} \cdot \Delta t \cdot \eta \quad \forall t$

$P_{\mathrm{B}, t}=P_{\mathrm{N}, t}+R_{t} \quad \Delta f_{t} \quad \forall t$

$P_{\text {min }}+R_{t} \leq P_{\mathrm{N}, t} \leq P_{\max }-R_{t} \quad \forall t$

$S o C_{\min }+0.5 R_{t} \leq S o C_{t} \leq S o C_{\text {max }}-0.5 R_{t} \quad \forall t$

$R_{t} \geq 0 \quad \forall t$. 


\section{Sample Average Approximation:}

$\min -C_{\mathrm{R}} \sum_{t=0}^{H-1} R_{t}+\mathbb{E}\left[\sum_{\mathrm{t}=0}^{\mathrm{H}-1} \mathrm{C}_{\mathrm{TOU}, \mathrm{t}} \mathrm{P}_{\mathrm{G}, \mathrm{t}}\right]$

s.t. $\quad P_{\mathrm{G}, t}=P_{\mathrm{L}, t}+P_{\mathrm{B}, t}+P_{\mathrm{pv}, t} \quad \forall t$

$S o c_{t+1}=S o C_{t}+P_{\mathrm{B}, t} \cdot \Delta t \cdot \eta \quad \forall t$

$P_{\mathrm{B}, t}=P_{\mathrm{N}, t}+R_{t} \quad \Delta f_{t} \quad \forall t$

$P_{\min }+R_{t} \leq P_{\mathrm{N}, t} \leq P_{\max }-R_{t} \quad \forall t$

$S_{0} C_{\min }+0.5 R_{t} \leq S o C_{t} \leq S o C_{\text {max }}-0.5 R_{t} \quad \forall t$

$\min -C_{\mathrm{R}} \sum_{t=0}^{H-1} R_{t}+\frac{1}{\mathrm{~N}}\left[\sum_{\mathrm{i}=1}^{\mathrm{N}} \sum_{\mathrm{t}=0}^{\mathrm{H}-1} \mathrm{C}_{\mathrm{TOU}, \mathrm{t}} \mathrm{P}_{\mathrm{G}, \mathrm{t}}^{(\mathrm{i})}\right]$

$R_{t} \geq 0 \quad \forall t$.

s.t. $\quad P_{\mathrm{G}, t}^{(i)}=P_{\mathrm{L}, t}+P_{\mathrm{B}, t}^{(i)}+P_{\mathrm{pv}, t} \quad \forall i, \forall t$

$S o C_{t+1}^{(i)}=S o C_{t}^{(i)}+P_{\mathrm{B}, t}^{(i)} \cdot \Delta t . \eta \quad \forall i, \forall t$

$P_{\mathrm{B}, t}^{(i)}=P_{\mathrm{N}, t}^{(i)}+R_{t} \Delta f_{t}^{(i)} \quad \forall i, \forall t$

$P_{\text {min }}+R_{t} \leq P_{\mathrm{N}, t}^{(i)} \leq P_{\text {max }}-R_{t} \quad \forall i, \forall t$

$S o C_{\text {min }}+0.5 R_{t} \leq S o C_{t}^{(i)} \leq S o C_{\text {max }}-0.5 R_{t} \quad \forall i, \forall t$

Averaged value on $\mathrm{N}$ scenarios

$R_{t} \geq 0 \quad \forall t$. 


\section{Agenda}

1 Context of the work

2 Problem formulation

3 Out-of-sample simulations

$4 \quad$ Closed-loop simulations

5 Conclusions \& future work 


\section{Impact of the number of scenarios:}

- Solve the planning problem several times with different numbers of scenarios $(\mathrm{N}=5,400,1000)$

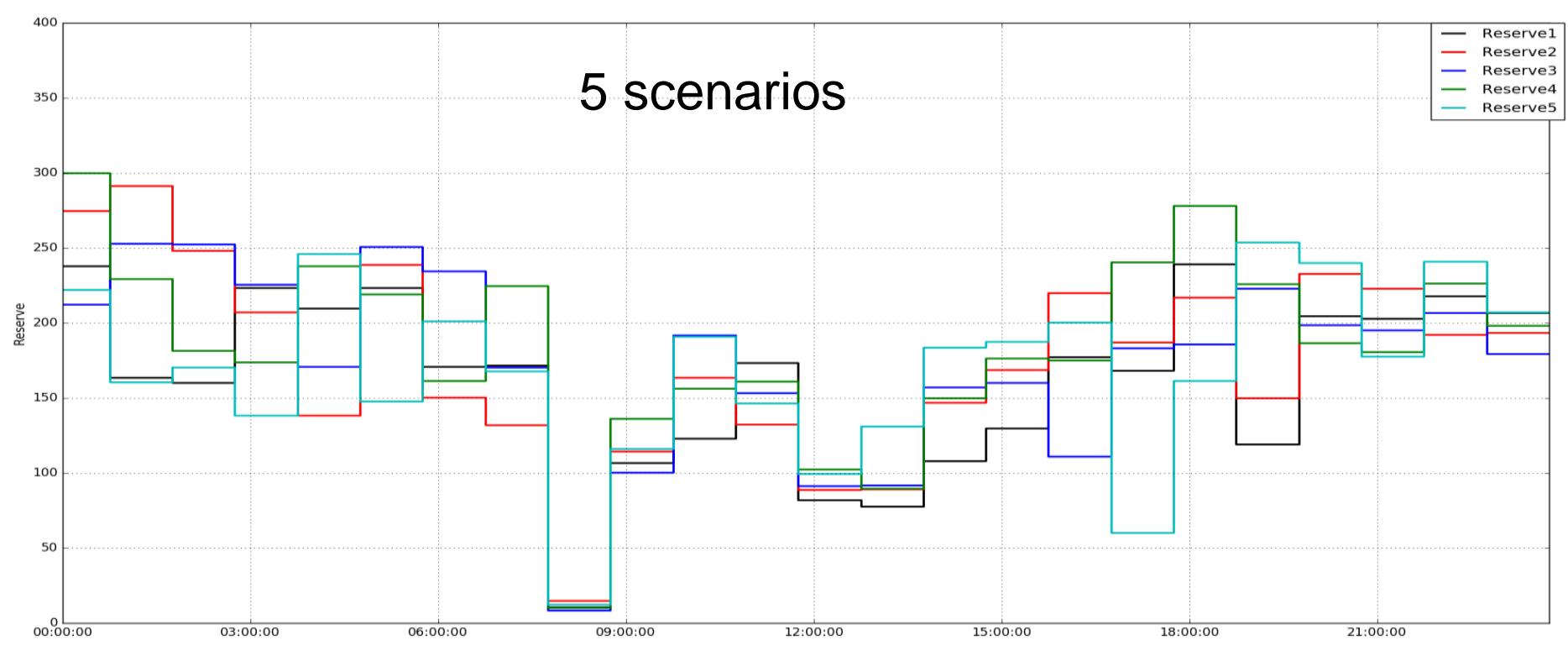




\section{Impact of the number of scenarios:}

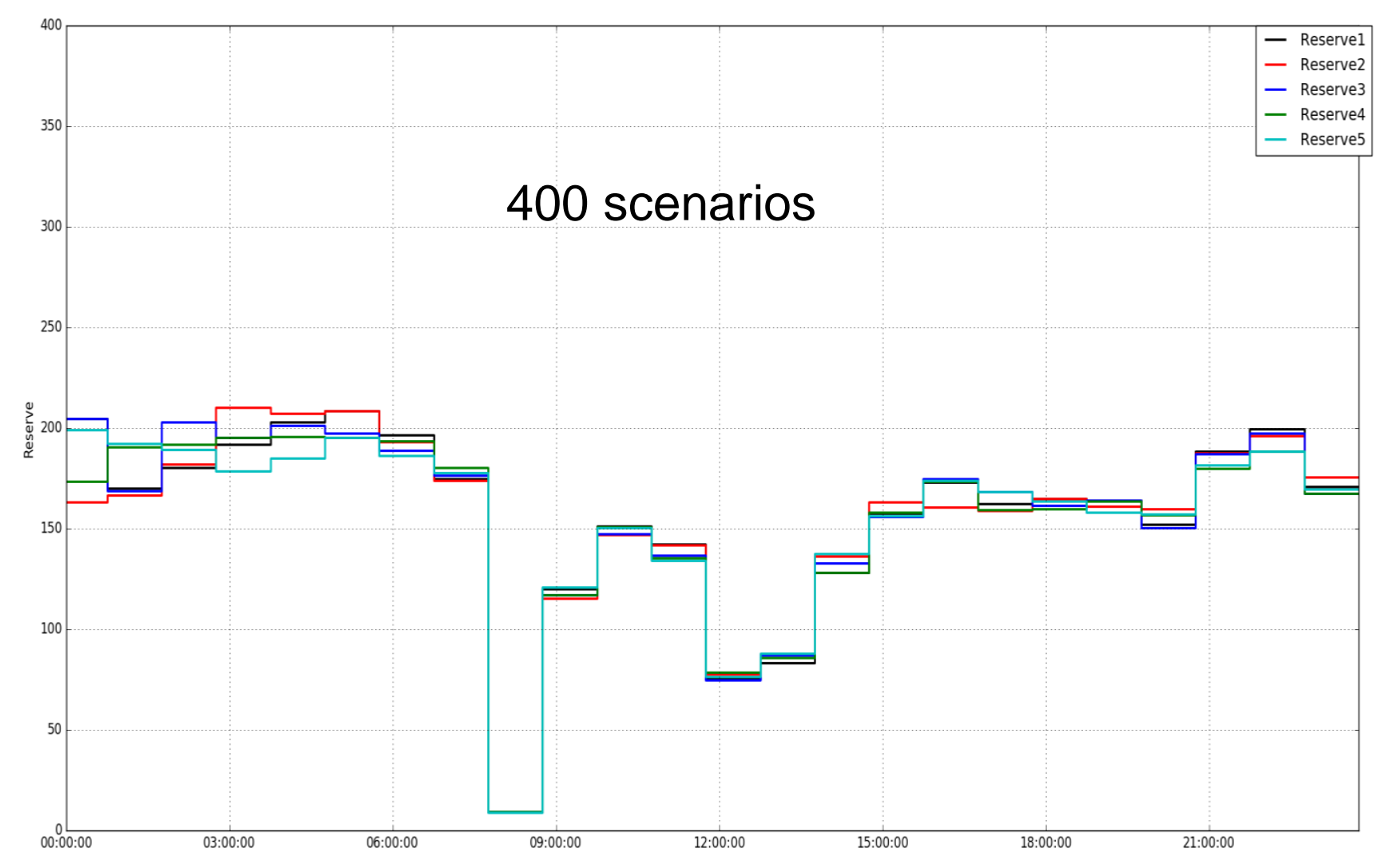




\section{Impact of the number of scenarios:}

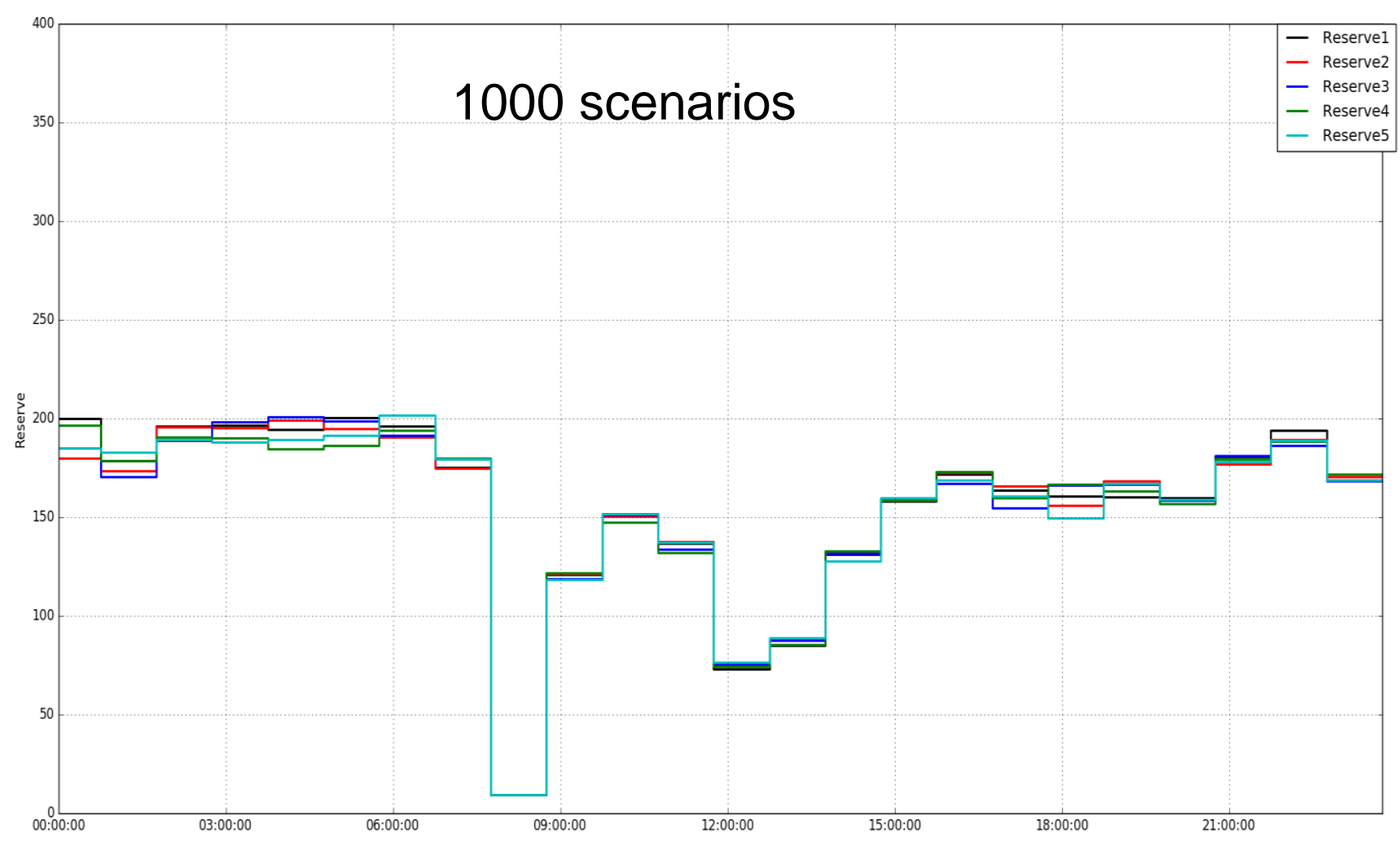




\section{Out-of-sample simulations:}

$N=5$ Solve several times the same planning problem

1

$N=400$
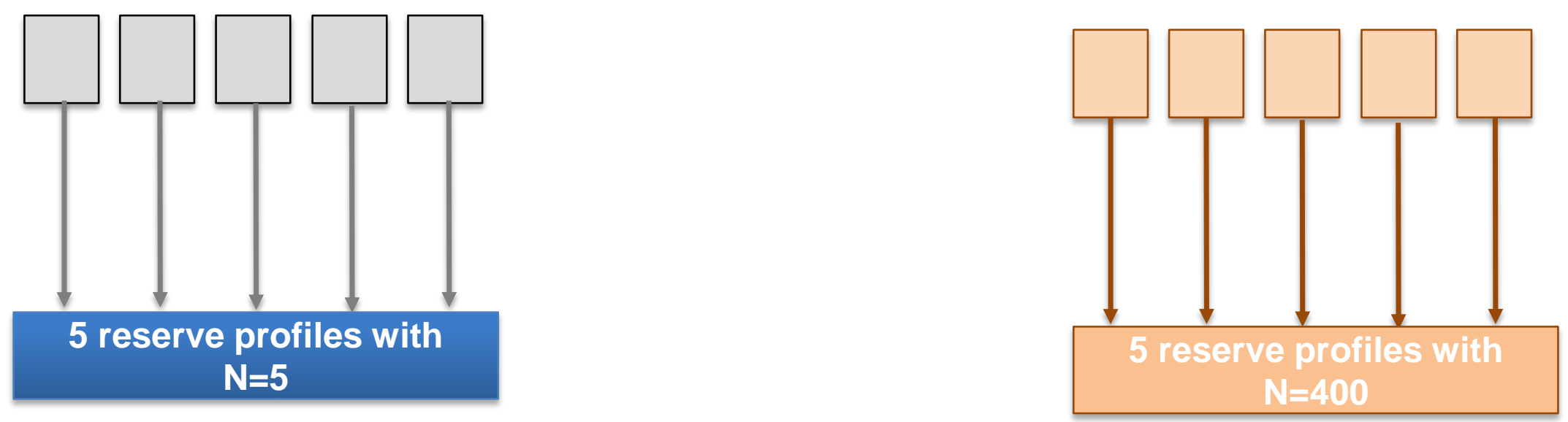

Generate another independent sample of 1000 scenarios

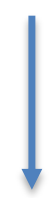

Solve 1000 variants of the control problem for each of the 10 reserve profiles
Compute the average of the 1000 obtained costs 


\section{Out-of-sample simulations:}

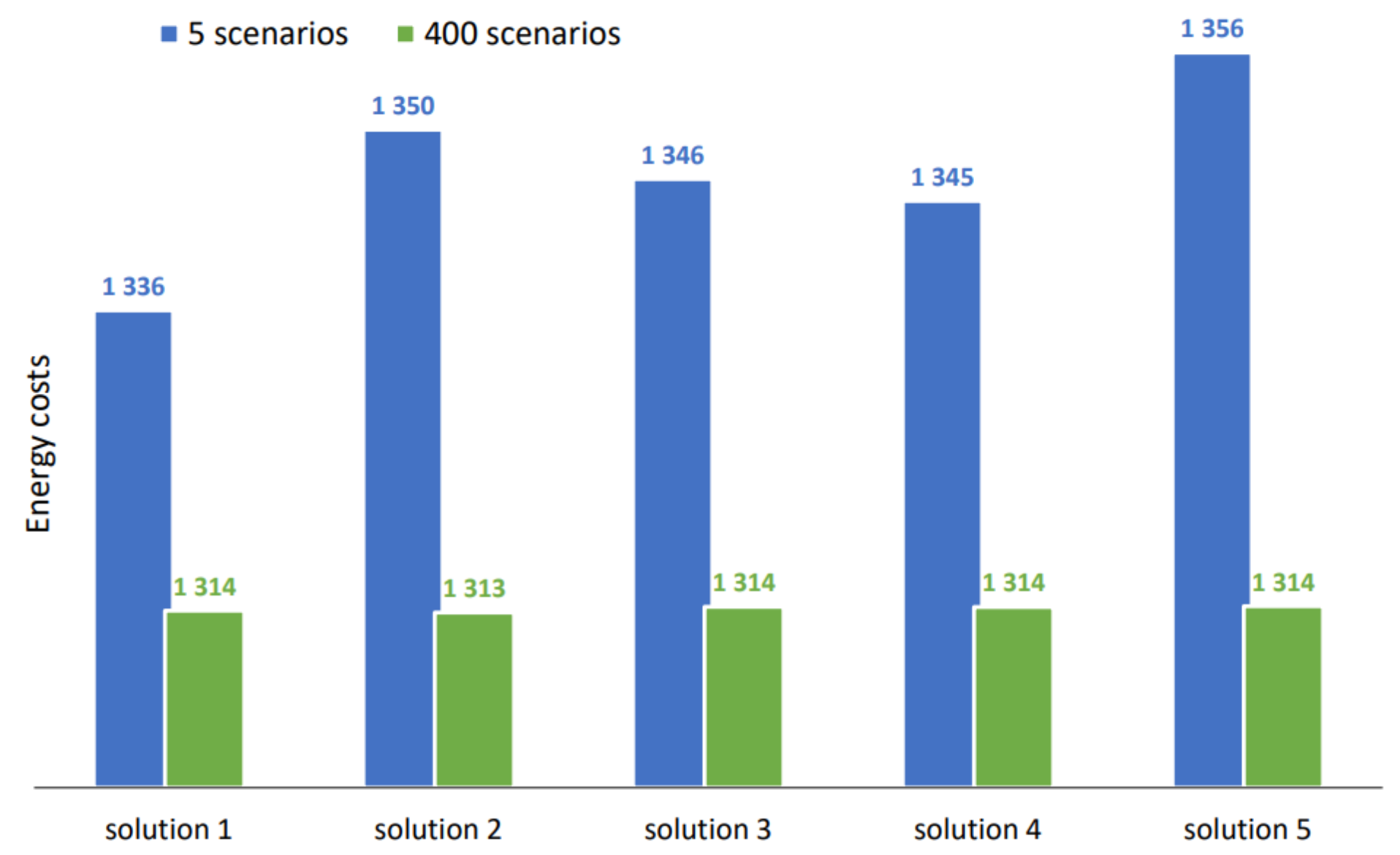

Page 17
5 


\section{Agenda}

1 Context of the work

2 Problem formulation

3 Out-of-sample simulations

$4 \quad$ Closed-loop simulations

5 Conclusions \& future work

Page 18 


\section{Closed-loop simulations:}

forecast (PV, load, prices)

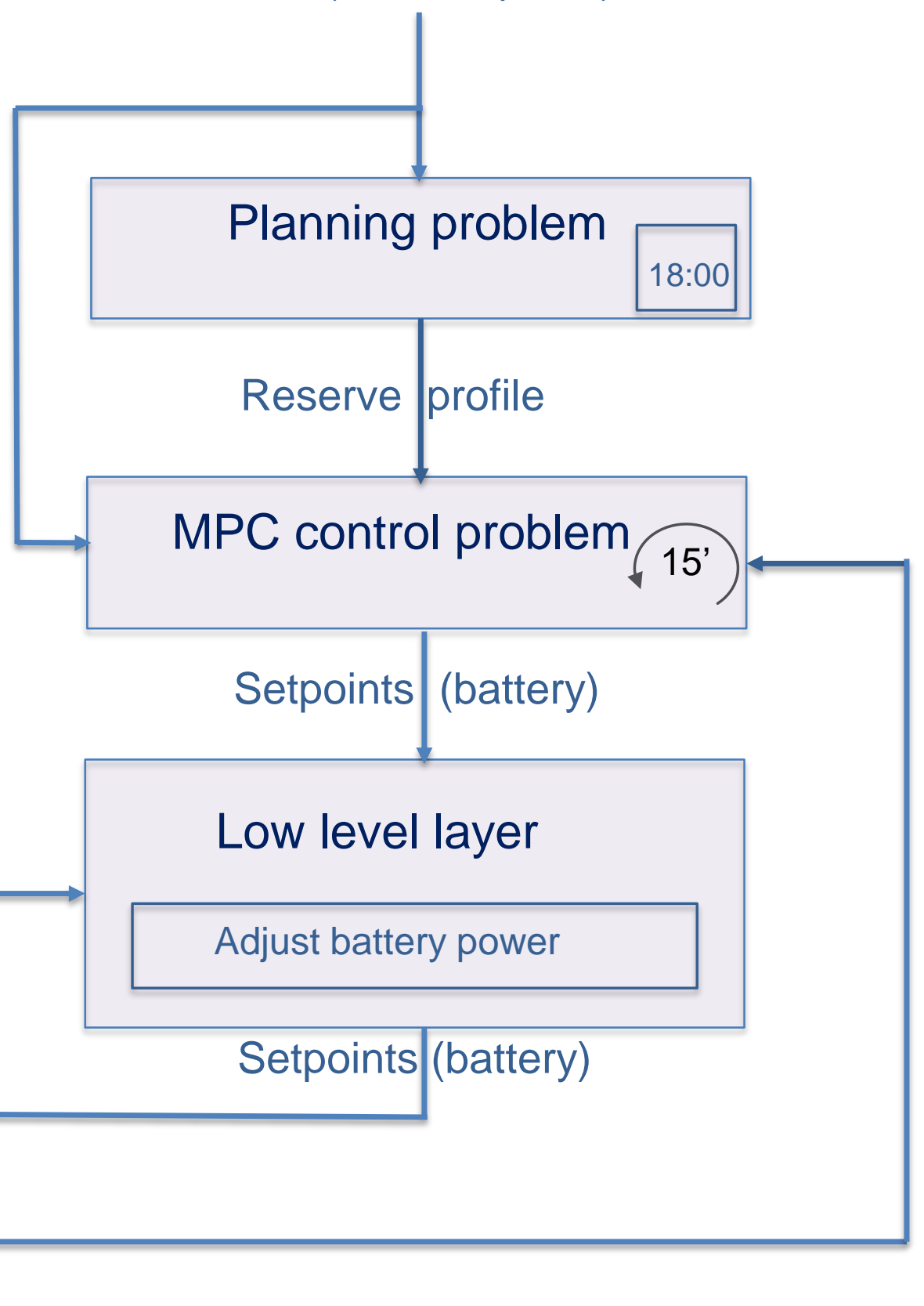

Page 19 
Closed-loop simulations: deterministic VS stochastic:

$$
\multimap \text { - deterministic }
$$

$$
-\infty-600 \text { scenarios }
$$

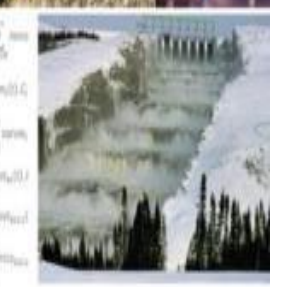

16,5

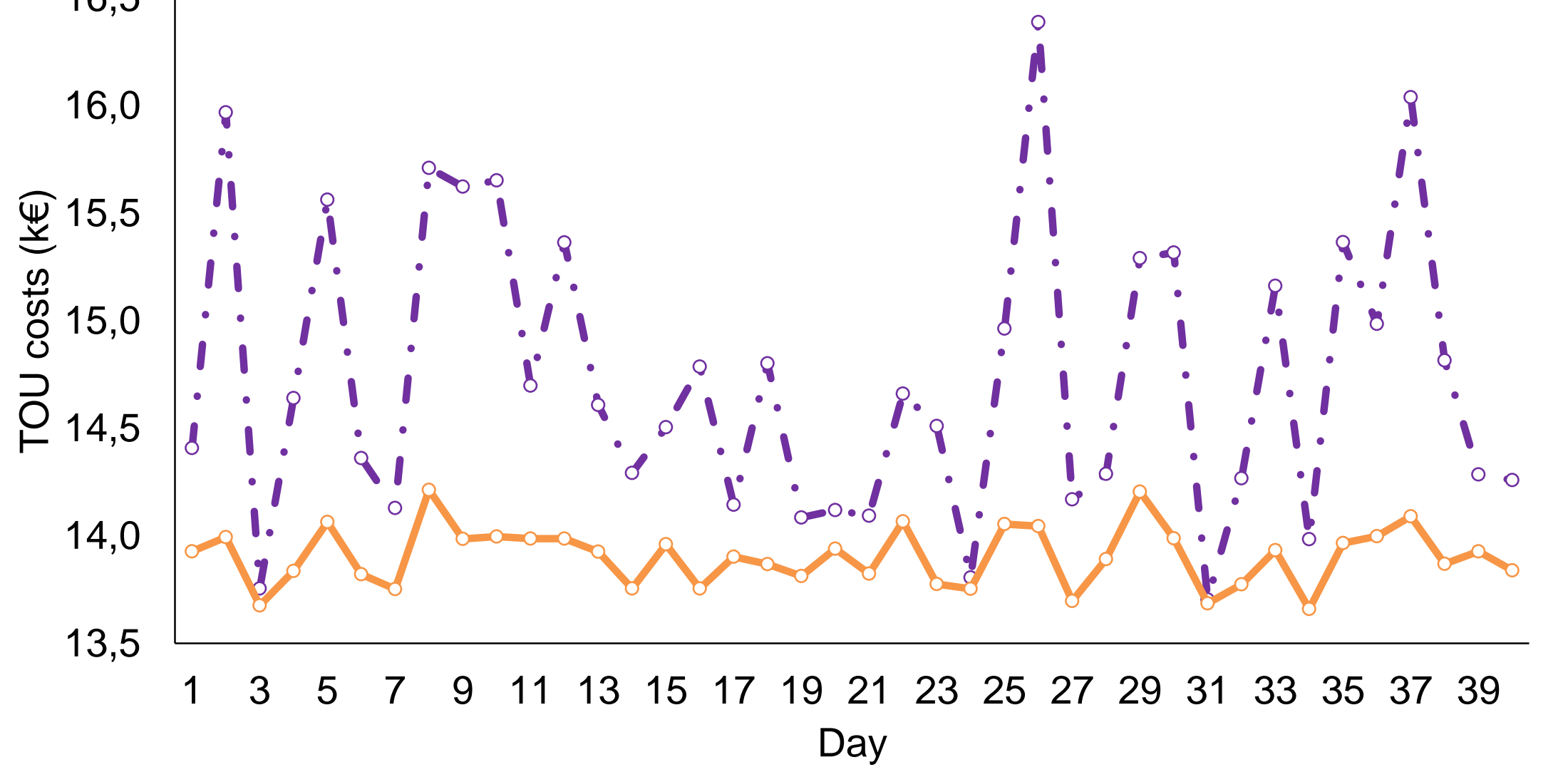


Closed-loop simulations: impact of the number of scenarios

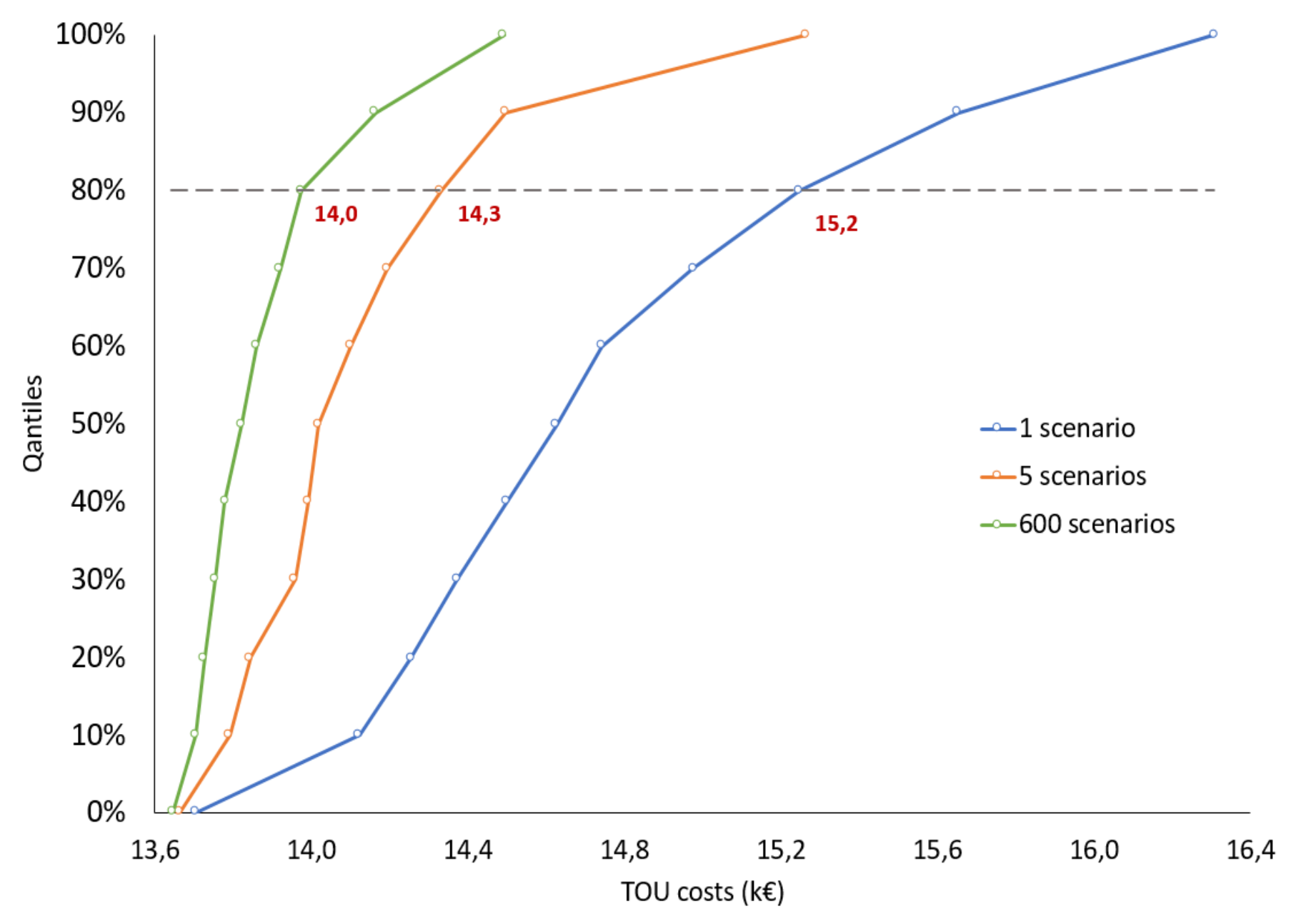

Page 21 


\section{Agenda}

1 Context of the work

2 Problem formulation

3 Out-of-sample simulations

4 Closed-loop simulations

$5 \quad$ Conclusions \& future work

Page 22

Life Is Un Schneider 


\section{Conclusion}

- Development of a stochastic programming approach to deal with the uncertainty in FCR

- Comparison with a classical/ deterministic approach

- Stochastic programming: reduced energy costs, reduced risks and a better predictability

\section{Future work}

- Reducing the computational time (decomposition/ clustering/ scenario reduction techniques...t

- Considering other sources of uncertainty ( load, PV production, electricity prices...)

- Extending to muli-energy optimization (thermal and electrical) 


\section{Thank you for your attention}
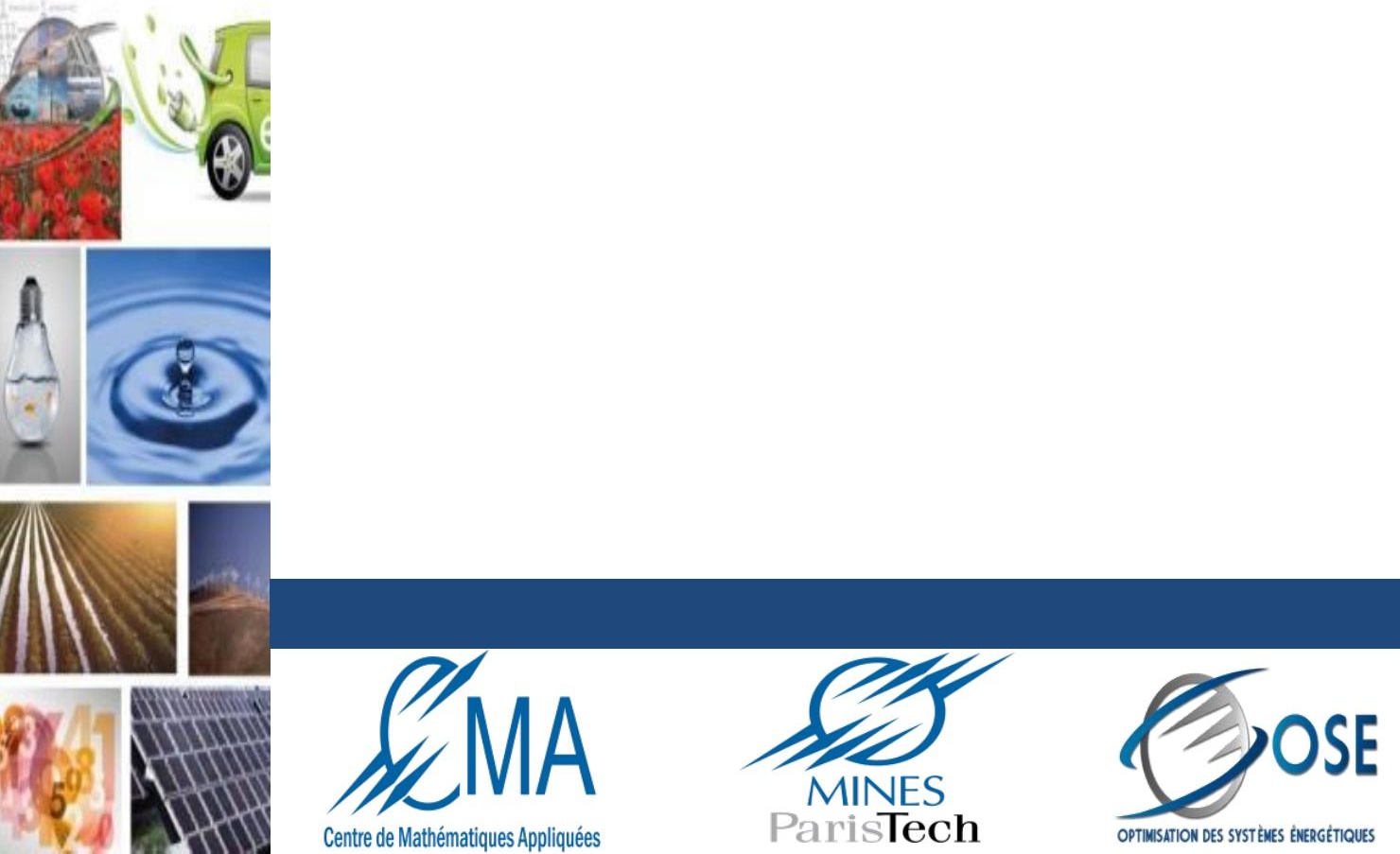

PSL $\star$

Schneider
$\mathbb{E} E$ Electric

ParisTech 
Closed-loop simulations: deterministic VS stochastic:

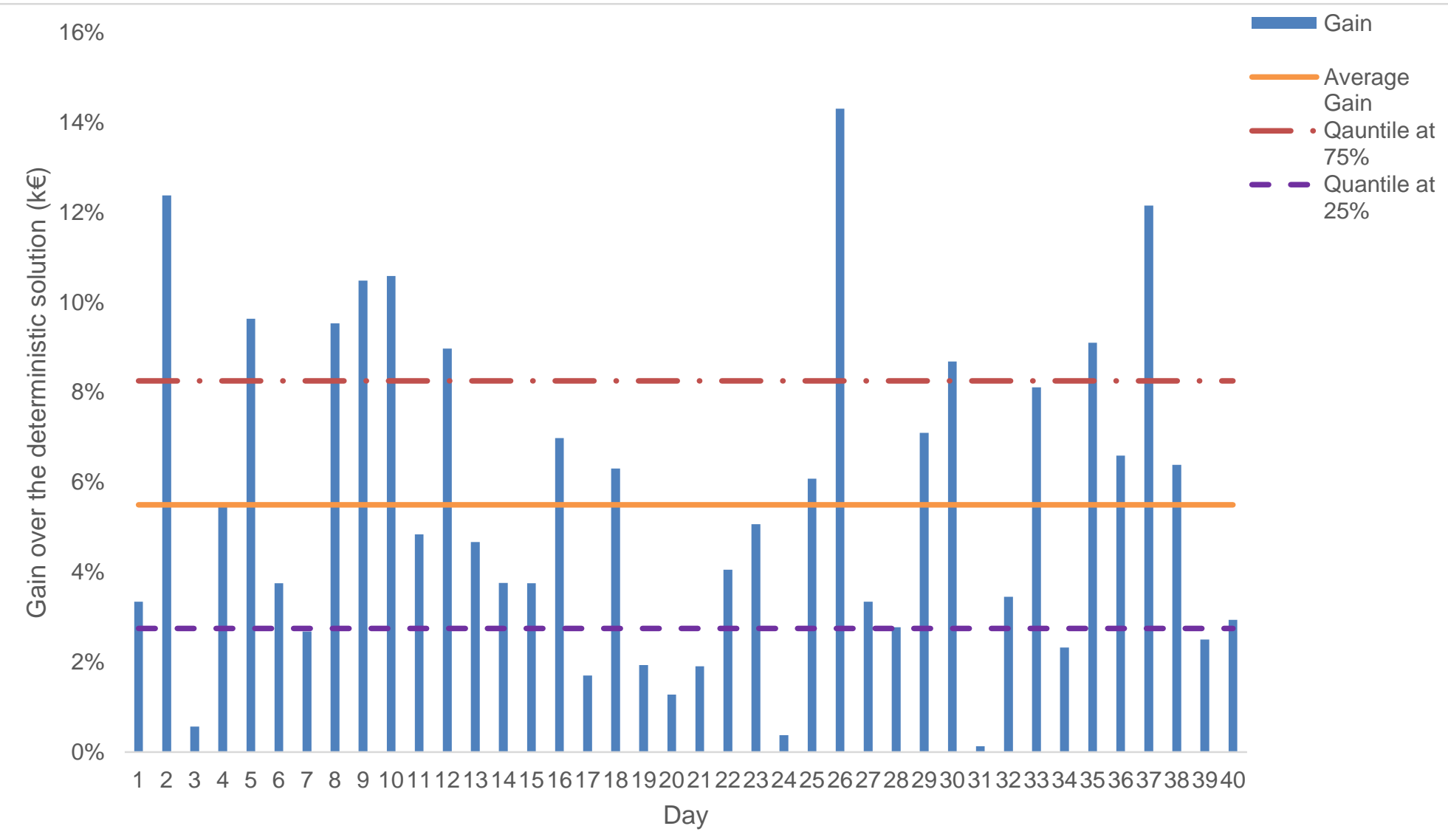

Page 25 vee rमfH 


\section{Closed-loop simulations: overview of the results}

Provide less frequency reserve to store surplus PV
No FCR reserve: battery used only for self-consumption

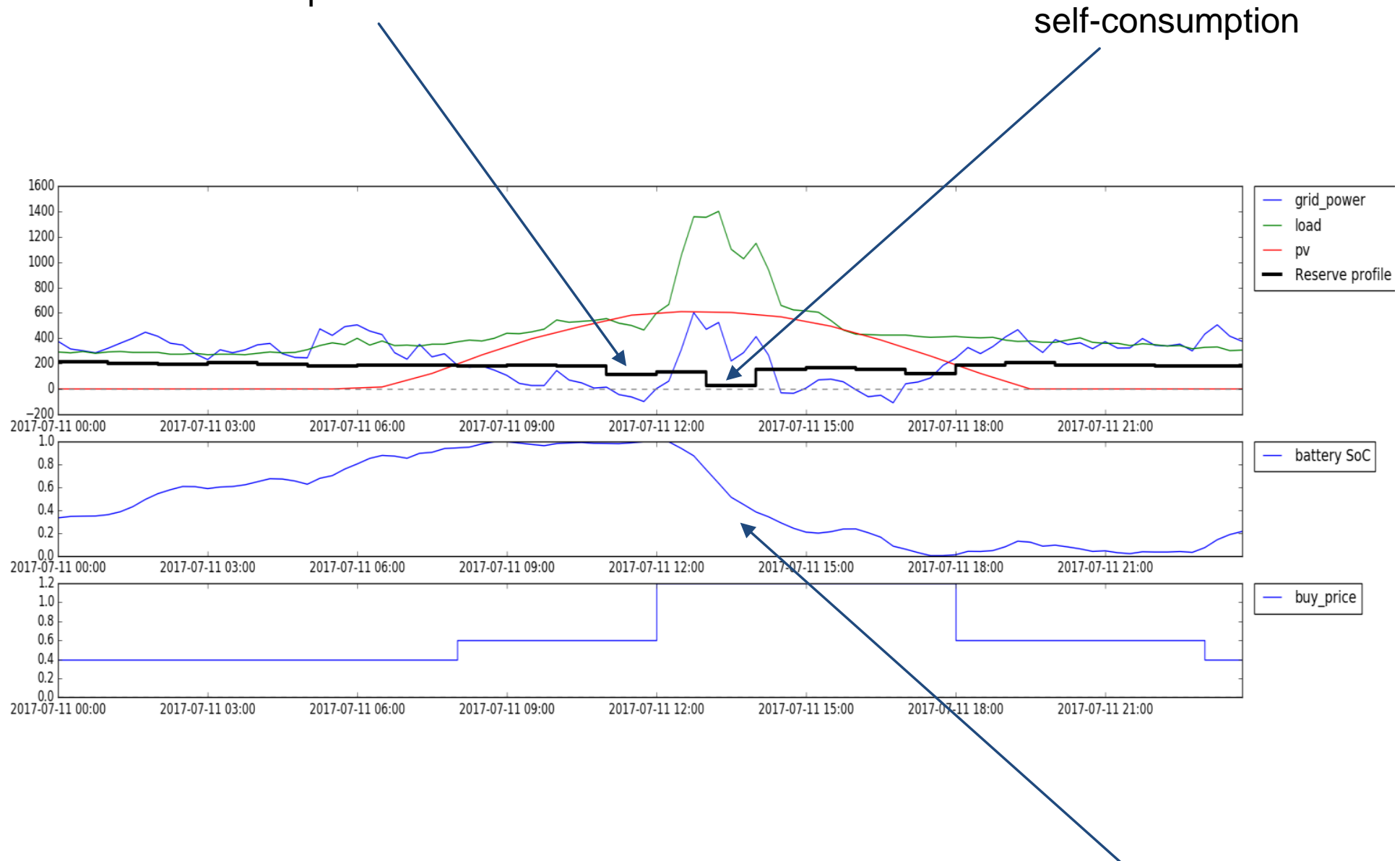

Discharging the battery during high price periods 